\title{
URINARY CHOLESTEROL EXCRETION IN MEN WITH BENIGN PROSTATIC HYPERPLASIA AND CARCINOMA OF THE PROSTATE
}

\author{
Dieter Juengst, MD, Angelika Pickel, Erich Elsaesser, MD \\ Franz J. Marx, MD, and Hans J. Karl, MD
}

\begin{abstract}
Urinary excretion of nonesterified (NEC) and total cholesterol (TC) has been investigated in 79 patients with prostatic adenoma (BPH) and 48 patients with carcinoma of the prostate. Normal range of NEC was determined in 62 healthy individuals and was found to be $0.26-2.2 \mathrm{mg} / 24$ hours $(2 \mathrm{SD})$ with a mean value of $0.76 \mathrm{mg} / 24$ hours. TC ranged from $0.3-2.9 \mathrm{mg} / 24$ hours with a mean value of $0.92 \mathrm{mg} / 24$ hours. In benign prostatic hyperplasia normal values for NEC and TC were determined in stages without residual urine. In contrast patients with BPH and residual urine showed elevated NEC in $42.1 \%$ and elevated TC in $61.4 \%$. In prostatic carcinoma NEC hyperexcretion was present in $44.8 \%$ of stage $A$ and $B$ and in $52.6 \%$ of stage $C$ and $D$. TC was elevated in $52.2 \%$ and $63.2 \%$, respectively. Simultaneous determination of NEC and serum acid phosphatase (SAP) gave a pathologic result of at least one parameter in $51.7 \%$ of stage $A$ and $B$ and in $84.2 \%$ of stage $C$ and $D$. If TC and serum acid phosphatase were combined, percentage of elevated values increases to 62.1 of stage $A$ and $B$ and was identical with 84.2 of stage $C$ and D. If BPH with residual urine and other known conditions of urinary cholesterol hyperexcretion in males, pluripotential malignant testicular neoplasms and diseases of kidney and urinary tract, can be excluded with reasonable certainty, both parameters may be of value in diagnosis of prostatic cancer in even early clinical stages of the disease, especially in combination with the determination of SAP.
\end{abstract}

Cancer 43:353-359, 1979.

$\mathrm{H}$ YPEREXCRETION OF URINARY NONESTERIFIED (NEC) or total cholesterol (TC) in prostatic cancer has been reported recently. ${ }^{3,11}$ It was concluded that both parameters are valuable in the diagnosis of the disease. A comparative evaluation of NEC and $\mathrm{TC}$ has not yet been undertaken; in addition, the performed studies did not show the value of these parameters in diagnosis of early clinical stages. Other known common conditions of urinary cholesterol hyperexcretion in males are pluripotential testicular neoplasms, diseases of kidney and urinary tract and benign prostatic hyperplasia. ${ }^{3,12,13,18,19}$ In regard to the frequency of $\mathrm{BPH}$ in comparison to prostatic carcinoma, it was additionally necessary

From the 2nd Medical Clinic München-Großhadern, the Urological Clinic of the University of Munich and the Department of Urology, Krankenhaus der Barmherzigen Brüder München, Munich, GFR.

Address for reprints: D. Juengst, MD, 2nd Medical Clinic, University Clinic München-Großhadern, Marchioninistr. 15, 8000 München 70, West Germany.

Accepted for publication March 15, 1978. to investigate whether elevated levels of urinary cholesterol were present in all clinical stages of BPH or only in patients with $\mathrm{BPH}$ and residual urine with indication for surgical treatment.

\section{Materials and Methods}

Nonesterified and total urinary cholesterol were analyzed in $2 \mathrm{ml}$ aliquots of 24-hour urine. After extraction with $6 \mathrm{ml}$ ethyl acetate for 30 minutes in a rotating system, the urinary phase was removed. The ethyl acetate extract was purified with alkali $(2 \mathrm{ml} 0.1 \mathrm{~N}$ $\mathrm{NaOH}$ ) and water washings and dried under a stream of nitrogen. The residue was dissolved with $0.1 \mathrm{ml}$ of the internal standard solution (10 mg 4-androstene-3, 17-dione/dl isooctane), followed by GLC on a $180 \mathrm{~cm} 1 \%$ XE $60 \mathrm{col}$ umn, i.d. $2 \mathrm{~mm}$, temp. const. 220 C. Quantitation was performed due to the peak height ratio, since alterations of cholesterol and 4androstenedione concentration gave a linear response. 

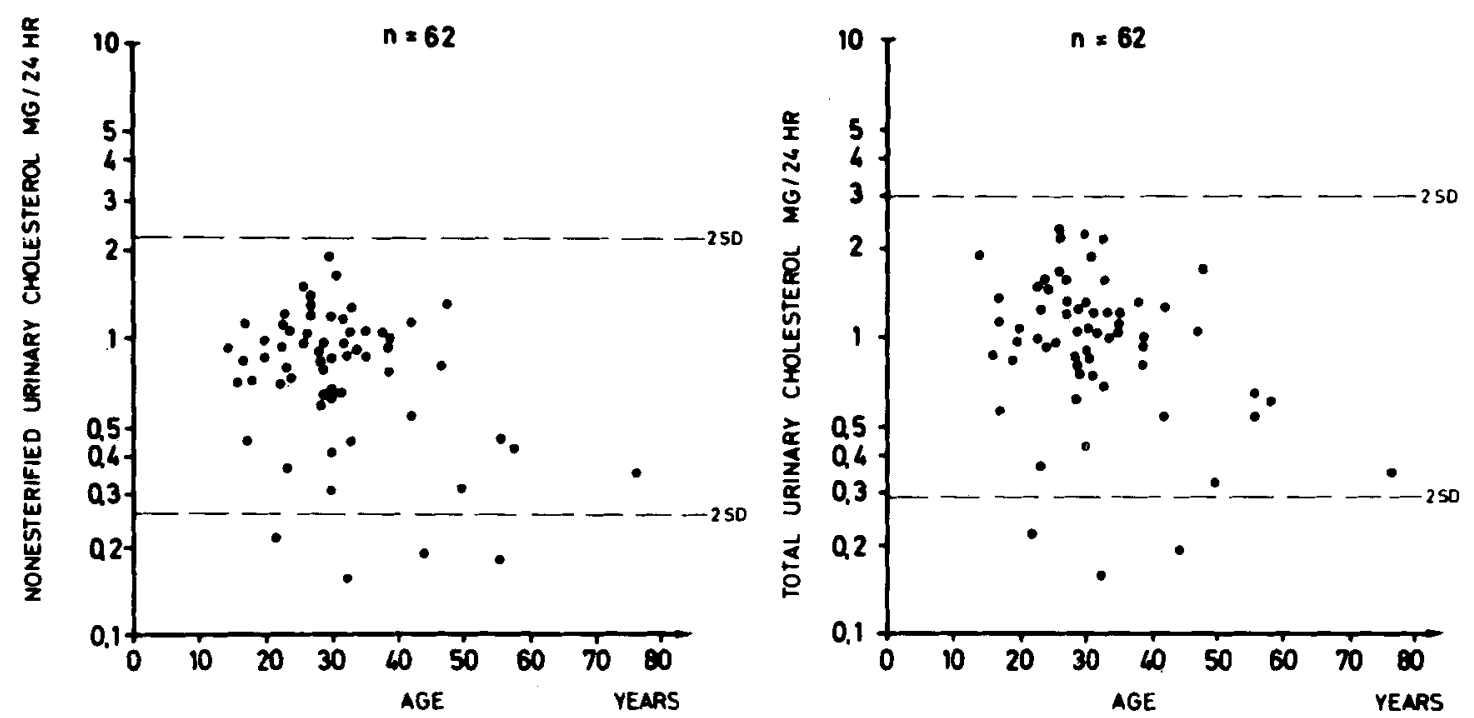

Fic. 1. Urinary excretion of nonesterified and total cholesterol in normal male individuals.

TG was measured as above including hydrolysis of the dried extract with $0.1 \mathrm{ml} \mathrm{3N}$ $\mathrm{KOH}$ and $0.9 \mathrm{ml}$ ethanol for 60 minutes at $60 \mathrm{C}$. NEC and TC were measured with an intraassay variance of $2.3 \%$ and $5.6 \%$ and an interassay variance of $11.2 \%$ and $10.9 \%$, respectively. Specificity of the method was confirmed by simultaneous masspectrographic analysis in selected samples.

\section{Clinical Material}

The clinical material consisted of patients with histopathologically proven prostatic neoplasms, which were divided in clinical stages $A-D$ according to Flocks. 11 patients were found to have a palpable prostatic nodule (stage $\mathrm{A}$ ); in 18 patients the prostatic cancer was not nodular but confirmed to one lobe of the gland (stage B). 5 patients showed local extension outside of prostate (stage C); in 14 cases metastasis in pelvis, other bones or lung were present. When urinary cholesterol determinations were performed, patients did not receive any treatment. A diagnosis of prostatic cancer had been established in most cases at the time of the investigation.

22 patients with BPH were diagnosed by rectal examination; they were without any symptoms (stage 0 ) or showed pollakiuria and impaired urinary stream (stage 1). Residual urine was excluded in all these cases. In 57 patients with $\mathrm{BPH}$ diagnosis was histopathologically proven after transurethral resection In all these patients residual urine was pres- ent (stage 2); in some combined with urinary incontinence or secondary kidney damage (stage 3).

The normal range for NEC and TC excretion was calculated using standard procedures and was obtained from a group of clinically healthy and biochemically normal individuals.

\section{Results}

\section{Normals}

This group comprised 62 clinically healthy, asymptomatic and biochemically normal male individuals covering an age range of 14 to 76 years.

The excretion values for NEC and TC are illustrated in Fig. 1. Statistical analysis of the results obtained gave a mean excretion of $0.76 \mathrm{mg} / 24$ hours NEC and $0.92 \mathrm{mg} / 24$ hours TC. The range for normal values was calculated as the mean $\pm 2 \mathrm{sD}$ including $95 \%$ of the population. On the basis of these results, the upper normal limit of the urinary excretion of NEC was considered to be $2.2 \mathrm{mg} / 24$ hours and for TC to be $2.9 \mathrm{mg} / 24$ hours. Hyperexcretion in the male, therefore, was defined as any result exceeding this value. It is important to note that the values of excretion of this group presented a log normal distribution.

\section{Prostatic Hyperplasia}

The results obtained in the study of 79 patients with a diagnosis of adenomatous pros- 

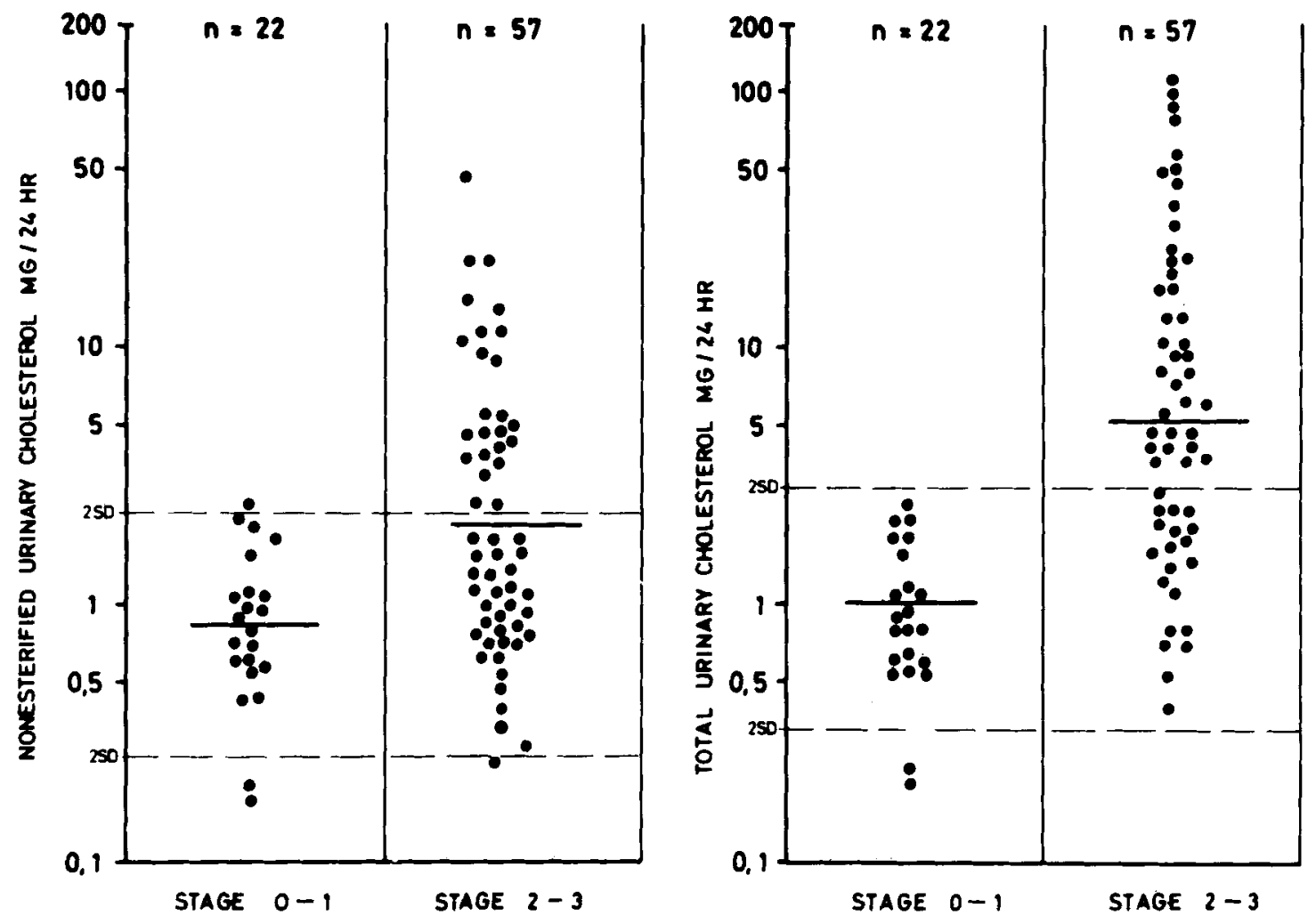

FIG. 2. Urinary excretion of nonesterified and total cholesterol in patients with a diagnosis of adenomatous prostatic hyperplasia.

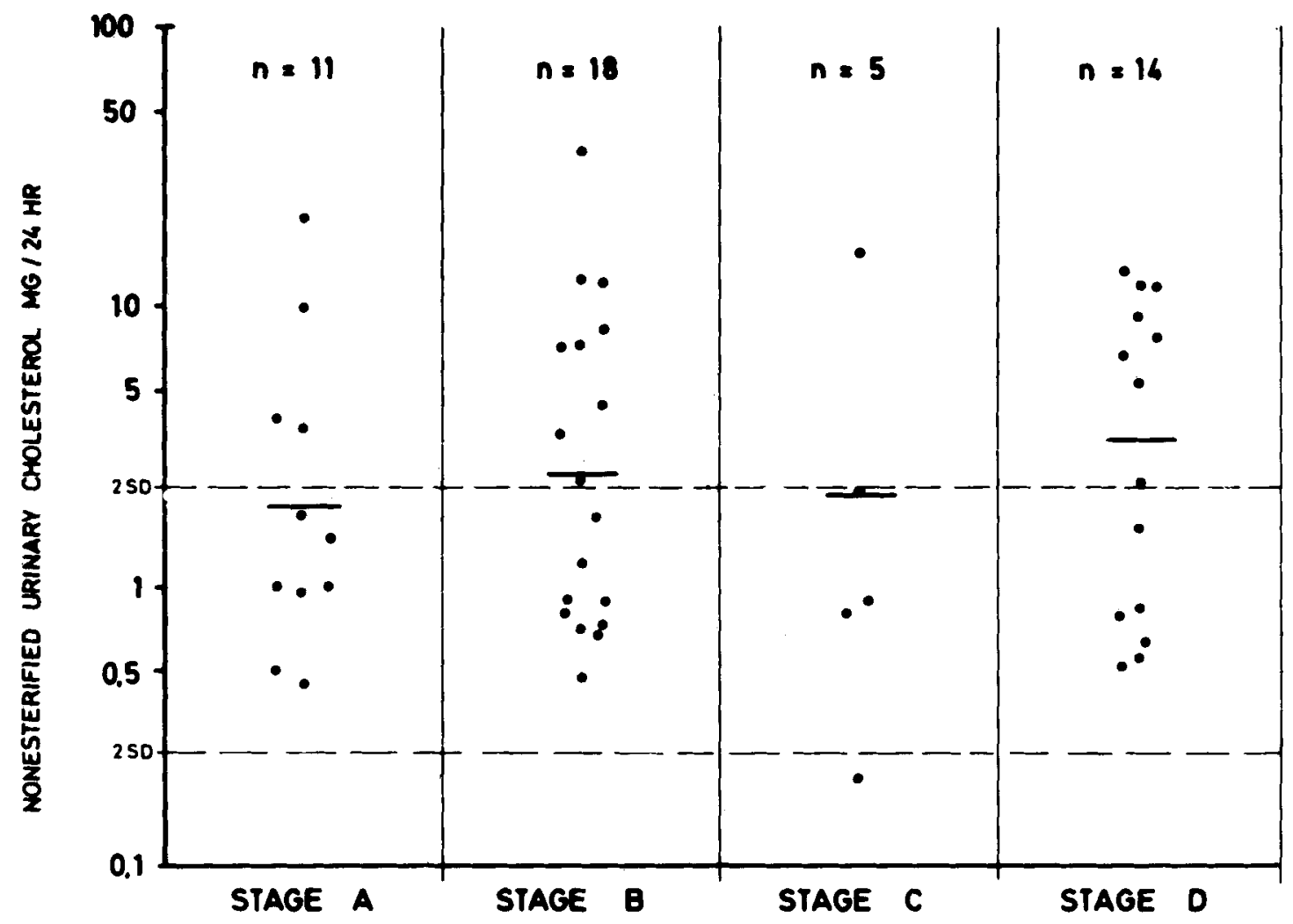

FIG. 3. Urinary excretion of nonesterified cholesterol in patients with prostatic carcinoma stage A-D. 


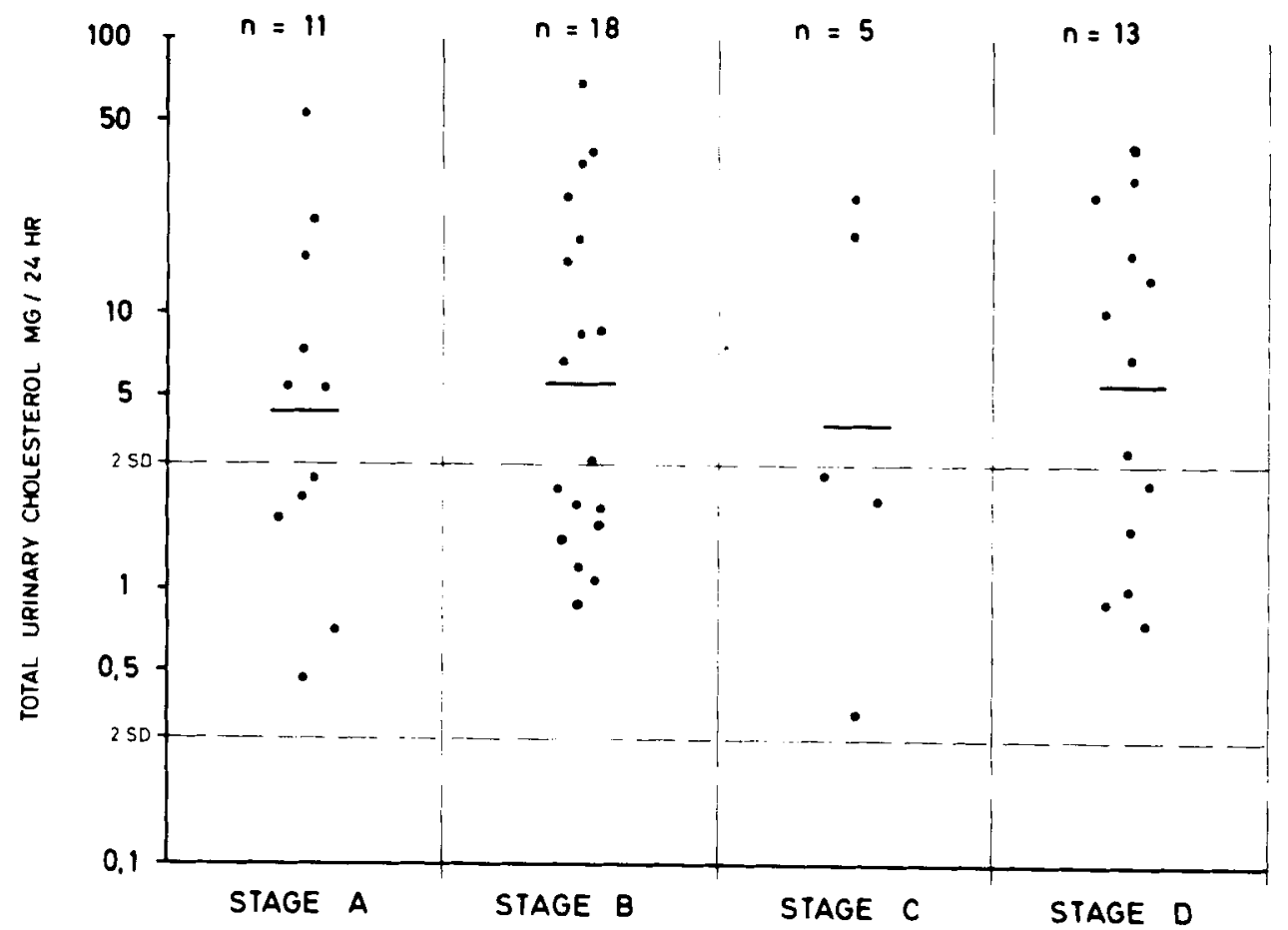

FIG. 4. Urinary excretion of total cholesterol in patients with prostatic carcinoma stage A-D.

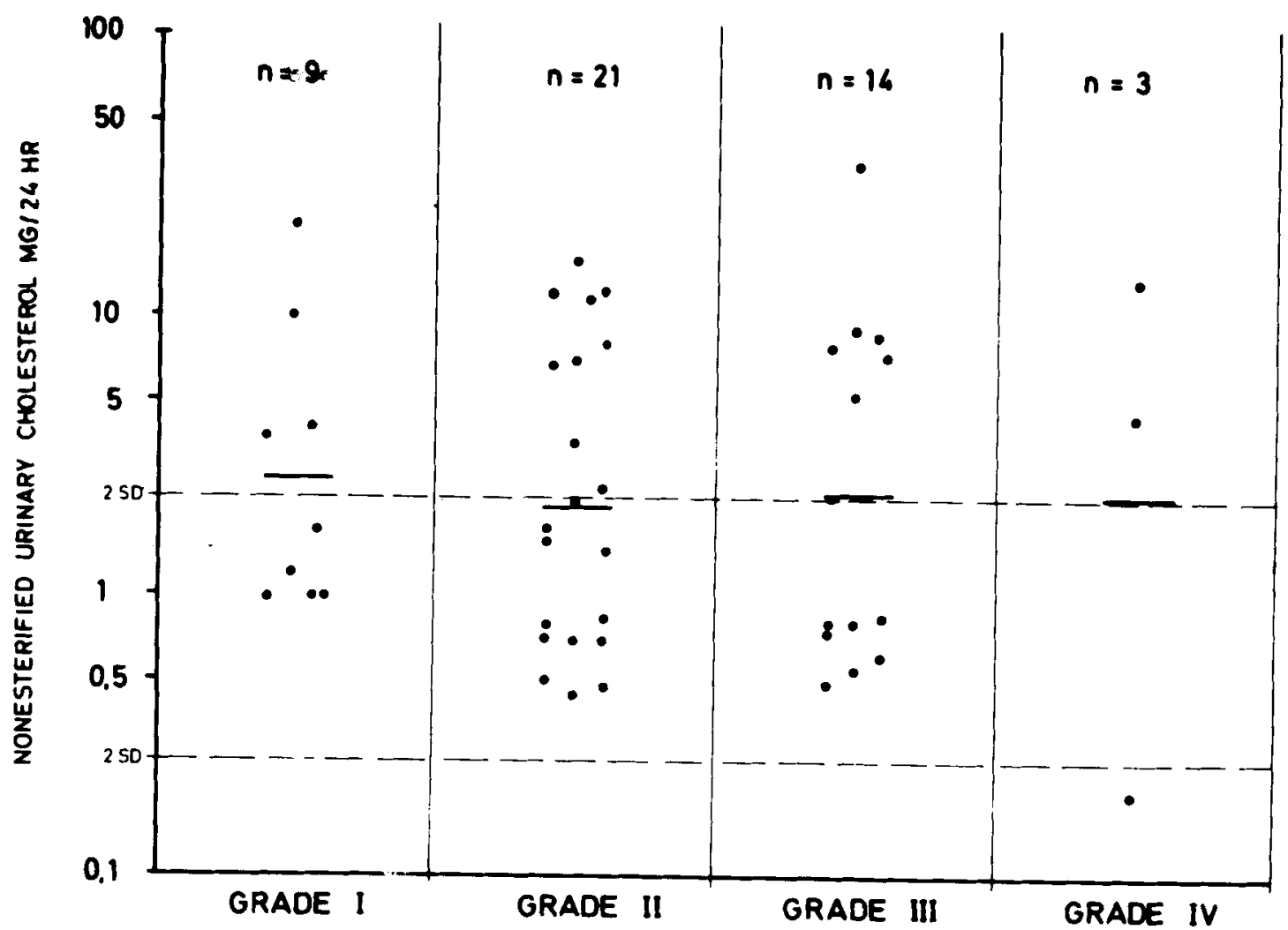

FIG. 5. Urinary excretion of nonesterified cholesterol in patients with prostatic carcinoma histological grade I-IV. 


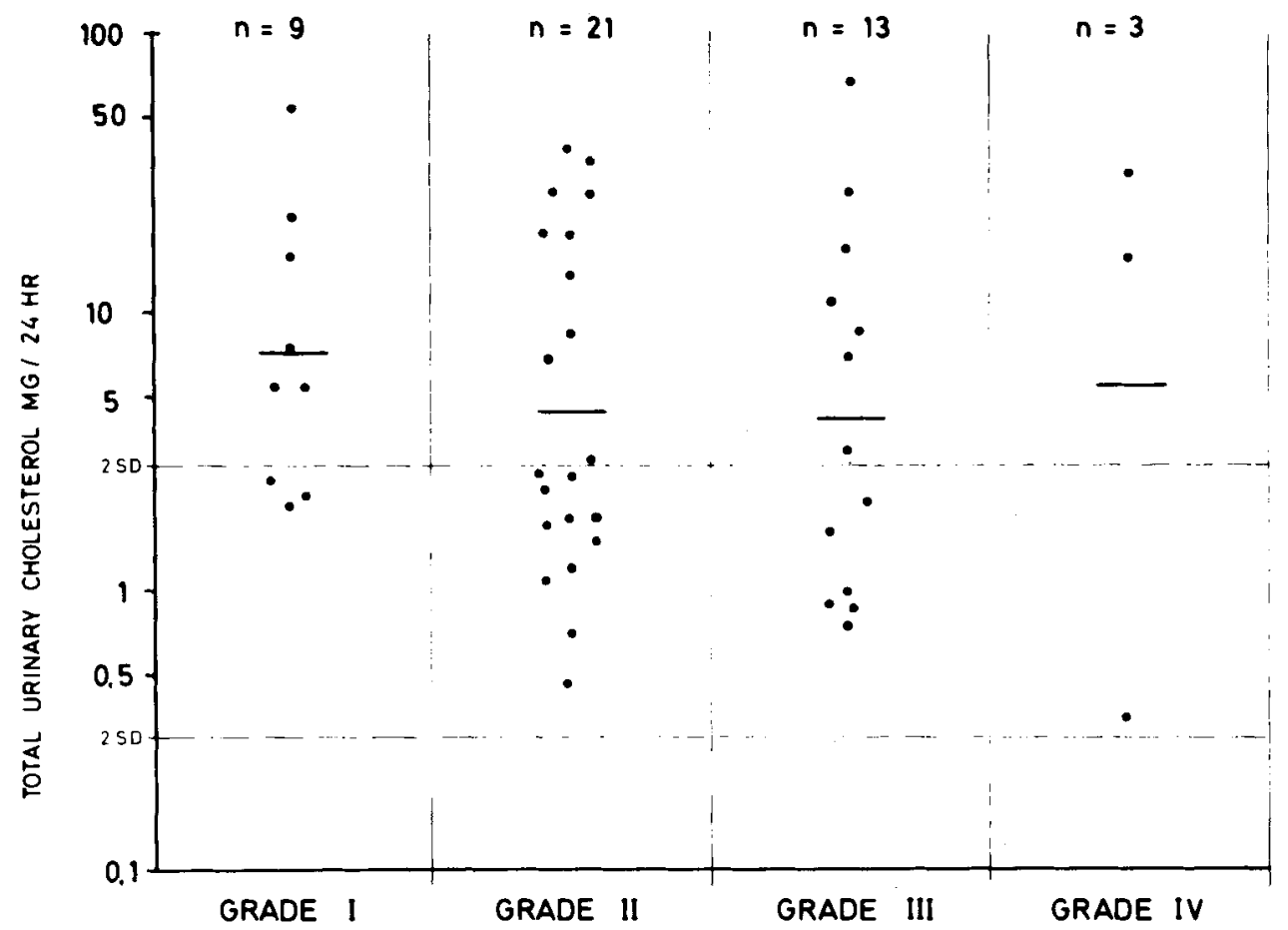

FIG. 6. Urinary excretion of total cholesterol in patients with prostatic carcinoma histological grade I-IV.

tatic hyperplasia are presented in Fig. 2. It is remarkable that in the 22 patients with stage $0-1$ NEC and TC values were normal in contrast to the 57 patients with stage $2-3$ who revealed elevated NEC in $42.1 \%$ and elevated TC in $61.4 \%$.

\section{Adenocarcinoma of the Prostate}

The NEC and TC values of 48 patients with prostatic carcinoma are illustrated in Figs. 36. The diagnosis was made on tissue obtained by needle biopsy or after resection. The group was divided in clinical stage $A-D$ according to Flocks (Figs. 3, 4) and based on the histologic examination in grade I-IV (Figs. 5, 6). Hyperexcretion of NEC was present in $44.8 \%$ of stage $A$ and $B$ and in $52.6 \%$ of stage $C$ and $\mathrm{D}$; elevated $\mathrm{TC}$ values were detected in $52.2 \%$ and $63.2 \%$ respectively.

Both parameters did not show any correlation to histological findings. It was remarkable that even in stage A NEC and TC hyperexcretion occurred; in all of these patients levels of serum acid phosphatase were normal.

Simultaneous determination of NEC and serum acid phosphatase gave a pathological result of at least one parameter in $51.7 \%$ of stage $A$ and $B$ and in $84.2 \%$ of stage $C$ and $D$
(Fig. 7). If TC and serum acid phosphatase were combined, percentage of elevated values increased to 62.1 of stage $A$ and $B$ and was identical with 84.2 in stage $C$ and D (Fig. 8).

\section{Discussion}

The association of enhanced serum acid phosphatase activity with prostatic neoplasms is well known; unfortunately, it is not suitable for detection of early clinical stages. ${ }^{12,14}$ Elevation of urinary nonesterified cholesterol in patients with prostatic cancer was first reported by Acevedo et al..$^{2}$ In a series of 32 patients, in $29(91 \%)$ hyperexcretion of NEC was observed based on an upper normal limit of 1.2 $\mathrm{mg} / 24$ hours. Nonesterified cholesterol seemed to be the major component, representing about $70 \%$ of total urinary cholesterol, but only in a few cases have NEC and TC been determined simultaneously. In a comparative evaluation of serum acid phosphatase and total urinary cholesterol in prostatic cancer, Chu et al. ${ }^{9}$ found pathologic values of serum acid phosphatase (SAP) in $67 \%$ and of urinary cholesterol in $62 \%$. When a combined determination of these two assays was performed, in $86 \%$ of the patients with prostatic cancer at least one parameter was elevated. 


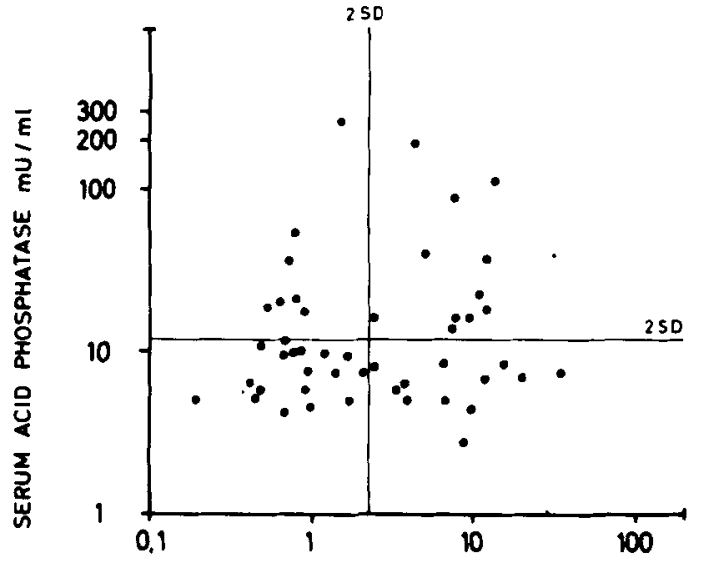

NONESTERIFIED URINARY CHOLESTEROL MG/24 HR

Fig. 7. Comparable evaluation of serum acid phosphatase and nonesterified urinary cholesterol in prostatic carcinoma.

It was concluded that a simultaneous determination of these parameters is valuable in diagnosing patients with prostatic neoplasia. However, it has to be mentioned that all patients except one had stage D carcinoma with diffuse osseous metastasis.

The percentage of pathologic results is dependent on the upper normal limit of an assay. For urinary nonesterified cholesterol we classified all values below $2.2 \mathrm{mg} / 24$ hours as normal. The higher percentage of elevated NEC values in prostatic carcinoma described by Acevedo et al. ${ }^{2}$ is caused by the lower upper

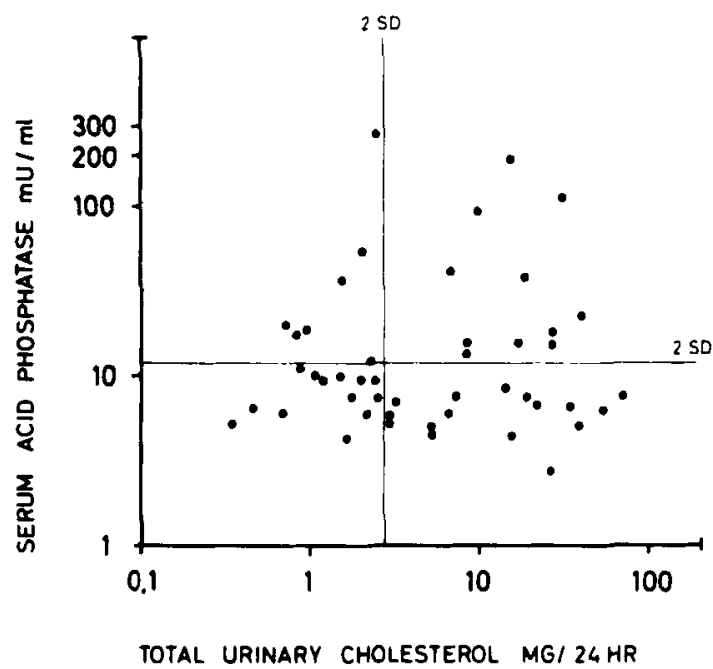

Fig. 8. Comparable evaluation of serum acid phosphatase and total urinary cholesterol in prostatic carcinoma. normal limit of $1.2 \mathrm{mg} / 24$ hours, which might be due to methodological differences. The rate of pathological urinary total cholesterol values in prostatic neoplasms reported by Chu et al. ${ }^{9}$ was very similar to those for stage $C$ and $D$ presented in this study, due to the nearly identical upper normal limit of $2.6 \mathrm{mg} / 24$ hours in comparison to $2.9 \mathrm{mg} / 24$ hours. Consequently we found in stage $\mathrm{C}$ and $\mathrm{D}$ a nearly identical rate of elevated values for simultaneous determination of SAP and total urinary cholesterol.

In benign prostatic hyperplasia stage $0-1$, urinary cholesterol values were normal. However, the presence of residual urine in $\mathrm{BPH}$ was associated in a considerable percentage with pathologic NEC and TC levels. In all the latter patients the diagnosis was done from tissue obtained by TLR, there was no evidence of cancer in the samples examined. The possibility of "occult" prostatic carcinoma being at least in part responsible for the observed pathologic urinary cholesterol levels could be not completely excluded, since such a diagnosis is done at autopsy and the complete gland has to be studied.

In discussing the clinical value of the NEC and TC test in the diagnosis of prostatic cancer, one has to consider that elevated urinary cholesterol levels had been detected in other diseases too, although the scientific value of older studies is limited due to the inadequate analytical techniques, as well as in some cases, to gross deficiency in patient selection. ${ }^{2,4,5,6,7}$, 8,9,10,12,17,18 Hyperexcretion of urinary cholesterol in males occurred in patients with pluripotential malignant testicular neoplasms and benign or malign diseases of kidney and urinary tract. ${ }^{3,12,13,18,19}$ If these diseases and $\mathrm{BPH}$ with residual urine can be excluded with reasonable certainty, NEC or TC determinations may be valuable in the diagnosis of prostatic cancer even in the early clinical stages of the disease, especially when in combination with serum acid phosphatase.

The physiologic mechanisms of urinary cholesterol excretion are not known; Acevedo et al. suggested an excretion as a protein bound complex. ${ }^{1}$ Hyperexcretion in nephrotic syndrome is possibly caused by increased glomerular filtration of plasma lipoproteins especially HDL fraction. ${ }^{15}$ If elevated urinary cholesterol is present without incidence of disease in the kidney and urinary tract, as in the case of prostatic carcinoma, higher production of specific lipoproteins by the malig- 
nant cells may be discussed. In patients with prostatic adenoma, the presence of residual urine could be responsible for the observed elevated urinary cholesterol values.

\section{REFERENCES}

1. Acevedo, H. F., and Campbell, E. A.: Urinary cholesterol III. Its excretion as a protein bound complex. Steroids 16:569-577, 1970 .

2. Acevedo, H. F., Campbell, E. A., Hayeslip, D. W., Gilmore, J., Merkow, L. P., Frich, J. C., Jr., and Grauer, R. C.: Urinary cholesterol IV. Its excretion in women with neoplasms of the genital system. Obstet. Gynecol. 37: 425-436, 1971 .

3. Acevedo, H. F., Campbell, E. A., Saier, E. L., Frich, J. C., Merkow, L. P., Hayeslip, D. W., Bartok, S. P., Grauer, R. C., and Hamilton, J. L.: Urinary cholesterol $V$. Its excretion in men with testicular and prostatic neoplasms. Cancer 32:196-205, 1973.

4. Acevedo, H. F., Campbell, E. A., Frich, I. C., Ir., Dugan, P. J., Saier, E. L., and Merkow, L. P.: Urinary cholesterol VI. Its excretion in women with inoperable inflammatory carcinoma of the breast. Cancer 34:17271736,1974

5. Acevedo, H. F., Campbell, E. A., Frich, J. C., Jr., Merkow, L. P., Hayeslip, D. W., and Gilmore, J.: Urinary cholesterol VII. The significance of the excretion of nonesterified cholesterol in patients with uterine carcinomas. Cancer 36:1459-1469, 1975.

6. Acevedo, H. F., Campbell, E. A., Frich, J. C., Hayeslip, D. W., and Gilmore, J.: Urinary cholesterol VIII. Its excretion in women with ovarian neoplasms. Cancer $37: 2847-2857,1976$

7. Bloch, E., and Sobotka, H.: Urinary cholesterol in cancer. J. Biol. Chem. 124:567-572, 1938.

8. Bruger, M., and Ehrlich, S. B.: Cholesterol content of the urine in patients with cancer. Arch. Intern. Med. 72:108-1 14, 1943

9. Burchell, M. M., Earle, J. H. O., and Maclagan, N. F.: Urinary cholesterol in cancer: Urinary cholesterol excretion in cancer patients and control subjects. $B r . J$. Cancer 3:42-51, 1949.
10. Burchell, M. M., and Maclagan, N. F.: Urinary cholesterol in cancer: Chemical state of urinary cholesterol and methods of estimation. Br.J. Cancer 3:52-61, 1949.

11. Chu, T. M., Shukla, S. K., Mittelman, A., and Murphy, G. P.: Comparative evaluation of serum acid phosphatase, urinary cholesterol and androgens in diagnosis of prostatic cancer. Urology 6:291-294, 1975.

12. Frick, J., and Spiteller, G.: Cholesterin und Harntrakterkrankungen. Zeitschr. Urol. 61:833-838, 1968.

13. Klahr, S., Tripathy, K., and Bolanos, O.: Qualitative and quantitative analysis of urinary lipids in the nephrotic syndrome.J. Clin. Invest. 16:1475-1481, 1967.

14. Kurtz, C. W., and Walk, W. L.: Limitations of prostatic acid phosphatase determination in carcinoma of prostate. J. Urol. 83:74-79, 1960.

15. De Mendoza, S. G., Kashyap, M. L., Chen, C. Y., and Lutmer, R. F.: High density lipoproteinuria in nephrotic syndrome. Metabolism 25:1143-11149, 1976.

16. Murphy, G. P., Reynoso, G., Kenny, G. M., and Gaeta, J. F.: Comparison of total and prostatic fraction serum acid phosphatase levels in patients with differentiated and undifferentiated prostatic carcinoma. Cancer 23:1309-1314, 1969 .

17. Sobotka, H., and Bloch, E.: Urine extractives in cancer. Am. J. Cancer 35:50-54, 1939.

18. Spiteller-Friedmann, M., Spiteller, G., Spiteller, H., and Frick, J.: Zur Frage der Ausscheidung von Cholesterin in Harn bei verschiedenen Erkrankungen (vornehmlich bei Tumorerkrankungen des Urogenitaltrakts). Öterr. Zeitschr. Krebsforsch. 5:25-35, 1971.

19. Zimmer, J. G., Dewey, R., Waterhouse, Ch., and Terry, R.: The origin and nature of anisotropic urinary lipids in the nephrotic syndrome. Ann. Intern. Med. 54: 205-214, 1961. 